\title{
ON THE SPECTRUM OF THE SYLVESTER-ROSENBLUM OPERATOR ACTING ON TRIANGULAR ALGEBRAS
}

\author{
L. W. Marcoux AND A. R. Sourour
}

Abstract. Let $\mathscr{A}$ and $\mathscr{B}$ be algebras and $\mathscr{M}$ be an $\mathscr{A}-\mathscr{B}$-bimodule. For $A \in \mathscr{A}, B \in \mathscr{B}$, we define the Sylvester-Rosenblum operator $\tau_{A, B}: \mathscr{M} \rightarrow \mathscr{M}$ via $\tau_{A, B}(M)=A M+M B$ for all $M \in$ $\mathscr{M}$. We investigate the spectrum of $\tau_{A, B}$ in three settings, namely: (a) when $\mathscr{A}=\mathscr{B}=\mathscr{T}_{n}(\mathbb{F})$, the set of upper-triangular matrices over an algebraically closed field $\mathbb{F}$ and $\mathscr{M} \subseteq \mathbb{M}_{n}(\mathbb{F})$; (b) when $\mathscr{A}=\mathscr{B}=\mathscr{M}$ is a unital triangular Banach algebra; and (c), when $\mathscr{M}=\mathscr{T}(\mathscr{N})$ is the nest algebra associated to a nest $\mathscr{N}$ on a complex, separable Hilbert space and $\mathscr{A}=\mathscr{B}=$ $\mathbb{C} I+\mathscr{K}(\mathscr{N})$ consists of the unitization of the algebra of compact operators in $\mathscr{T}(\mathscr{N})$.

Mathematics subject classification (2010): 15A06, 15A24, 46H25, 47L35.

Keywords and phrases: Sylvester equation, Sylvester-Rosenblum operator, triangular algebra, nest algebra.

\section{REFERENCES}

[1] W. ARendt, R. RÄBiger, AND A. Sourour, Spectral properties of the operator equation $A X+X B=Y$, Quart. J. Math. Oxford Ser., 45:133-149, 1994.

[2] R. Bhatia And P. Rosenthal, How and why to solve the operator equation $A X-X B=Y$, Bull. London Math. Soc., 29:1-21, 1999.

[3] Ju. L. DALecki, On the asymptotic solution of a vector differential equation, Dokl. Akad. Nauk. SSSR., 92:881-884, 1953.

[4] JU. L. DALECKI AND M.G. KReIn, Stability of solutions of differential equations in Banach space, volume 43 of Transl. Math. Monographs, Amer. Math. Soc., Providence, R.I., 1974.

[5] K.R. DAvidSON, Nest algebras. Triangular forms for operators on a Hilbert space, volume 191 of Pitman Research Notes in Mathematics, Longman Scientific and Technical, Harlow, 1988.

[6] J.A. ERdos AND S.C. POWER, Weakly closed ideals of nest algebras, J. Operator Theory, 7:219-235, 1982.

[7] D. LARSON, Triangularity in operator algebras, In Surveys of some recent results in operator theory, II, Pitman Res. Notes Math Ser. 192, pages 121-188, Harlow, 1988. Longman Sci. Tech.

[8] G. Lumer And M. Rosenblum, Linear operator equations, Proc. Amer. Math. Soc., 10:32-41, 1959.

[9] J.R. Ringrose, Super-diagonal forms for compact linear operators, Proc. London Math. Soc., 12:367-384, 1962.

[10] M.G. Rosenblum, On the operator equation $B X-X A=Q$, Duke Math. J., 23:263-270, 1956.

[11] J. Sylvester, Sur l'équation en matrices $p x=x q$, C. R. Acad. Sci. Paris, 99:67-71, 115-116, 1884. 